\title{
Computing Homographies from Three Lines or Points in an Image Pair ${ }^{\star}$
}

\author{
G. López-Nicolás, J.J. Guerrero, O.A. Pellejero, and C. Sagüés \\ DIIS - I3A, Universidad de Zaragoza. \\ C/María de Luna 1, E-50018 Zaragoza, Spain \\ \{gonlopez, jguerrer, csagues\}@unizar.es
}

\begin{abstract}
This paper deals with the computation of homographies from two views in a multi-plane scene. In the general case, homographies can be determined using four matched points or lines belonging to planes. We propose an alternative method when a first homography has been obtained, and then three matches are sufficient to compute a second homography. This process is based on the geometric constraint introduced by the first homography. In this work, the extraction and matching of features, points or lines, is automatically performed using robust techniques. Experimental results with synthetic data and real images show the advantages of this approach. Besides, the performance using points or lines as image features is compared.
\end{abstract}

Keywords: Homographies, multi-plane scenes, multi-view constraints, point and line matching.

\section{Introduction}

The algorithm we are going to describe deals with scenes containing planar surfaces. These are characteristic of urban scenes and other man made environments, in which lines and planes are plentiful elements. In these cases, the recovery of the $3 \mathrm{D}$ geometry can be supported by homographies computation.

Several authors detect and use planar homographies in image pairs [1], 2], but the detection of different homographies is considered as independent processes. There, the estimation of each homography is carried out independently of the others repeating the same algorithm each time.

The knowledge of one homography in an image pair impose restrictions on the other homographies. So the collection of homographies of multiple planes between two views spans a 4-dimensional linear subspace 3. However, this constraint requires the number of planes in the scene to be greater than four. Other works [4, 5] apply the constraint replacing the need for multiple planes by the need for multiple views. They only need two planes, and often a single one is enough under restricted assumptions on camera motion, but more than four views are necessary.

\footnotetext{
^ This work was supported by project DPI2003-07986.
} 
In previous works [6], we address the estimation of two or more homographies in an image pair and the computation of the fundamental matrix through them, using straight lines extracted and matched as described in [7. It has been reported that the multi-plane algorithm is not as stable as the general method to compute the fundamental matrix [1, but when less than three planes are observed, which is quite usual in man made environments, the multi-plane algorithm gives similar and even better results than the general method.

This work is focused in the case of two views. Usually, more than four planes are not available in the scene, so it is necessary to use different constraints from the ones presented in [5]. Here we present the computation of the second homography using the constraints imposed by a first one, through the homology of an image into itself. A complete algorithm which uses these constraints, matching lines or points automatically, is presented. Experimental results with synthetic data and real images show the advantages of this approach.

\section{Constraints on Homographies}

The computation of a homography from three point correspondences in an image pair knowing the fundamental matrix is presented in [8]. Now we show that a similar process can also be carried out, but using another homography instead of the fundamental matrix.

In order to compute a first homography $\mathbf{H}_{21}^{\pi_{1}}$ for a plane $\pi_{1}$ we need at least four matched points or lines belonging to that plane. Once this homography is obtained, a second homography $\mathbf{H}_{21}^{\pi_{2}}$ for a plane $\pi_{2}$ can be determined with only three points or lines using the constraints introduced by the first homography.

A useful method for real applications needs to be robust. We have chosen the RANSAC method, which is robust in presence of outliers. The presented approach reduces the sample size needed to have a minimum set of data required to instantiate the free parameters of the model. The number of samples necessary to ensure that at least one of them has no outliers depends on the sample size. Therefore, a shorter sample size leads us to a faster algorithm.

\subsection{Working with Points}

The method for computing a second homography from three points consists in obtaining a fourth point using the constraints provided by the first homography and the idea of plane parallax [8]. Once we get the set of four points, the general algorithm can be used to obtain the second homography.

Let us suppose the computed homography $\left(\mathbf{H}_{21}^{\pi_{1}}\right)$ induced by plane $\pi_{1}$ and the projections in both images $\left(\mathbf{p}_{1}, \mathbf{p}_{2}\right)$ of a point $(\mathbf{p})$ belonging to the second plane $\pi_{2}$ (Fig. 1 1 ). The corresponding of $\mathbf{p}_{1}$ through the homography $\mathbf{H}_{21}^{\pi_{1}}$ is $\overline{\mathbf{p}}_{2}=\mathbf{H}_{21} \mathbf{p}_{1}$. The epipolar line joining $\mathbf{p}_{2}$ to $\overline{\mathbf{p}}_{2}$ is the epipolar line of $\mathbf{p}$ in the second image. Repeating the process with another point $\mathbf{q}$, the line joining $\mathbf{q}_{2}$ to $\overline{\mathbf{q}}_{2}=\mathbf{H}_{21}^{\pi_{1}} \mathbf{q}_{1}$ is obtained. The intersection of these two lines determine the epipole $\mathbf{e}_{2}$. This is the fourth point used for the computation of the second homography. 


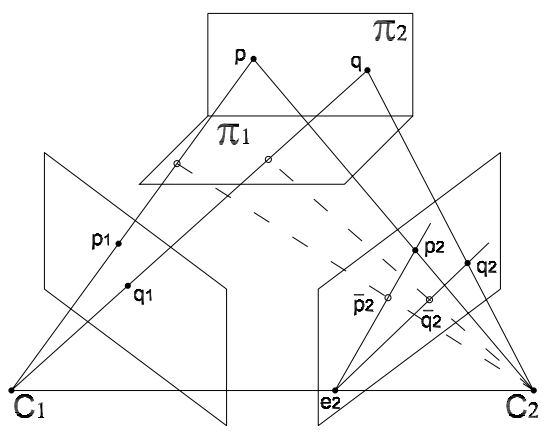

(a)

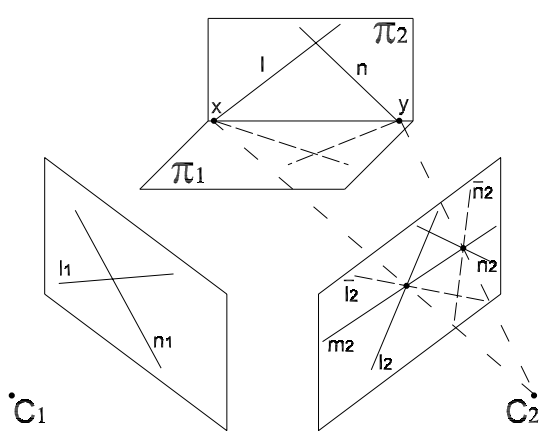

(b)

Fig. 1. Geometric method to obtain a second homography using points (a) or lines (b)

Two matched points are sufficient to get the epipole. As three points are available, three epipolar lines can be obtained. The intersection of these lines is the epipole, but they will not intersect exactly in a point due to image noise. So, the epipole is computed as the centroid of the triangle defined by these three lines.

A homography cannot be computed if three of the four correspondences are collinear. Therefore, the method for determining the second homography fails if one of the three points is the epipole or if two of the points are collinear with the computed epipole. Besides, when points are nearly collinear with the epipole, the method will give a poorly conditioned estimation of the homography.

\subsection{Working with Lines}

The process is similar using straight lines instead of points. Three lines are available, if we find the fourth line, a second homography will be determined.

Line $\mathbf{l}$ belonging to the plane $\pi_{2}$ is projected in both views as $\left(\mathbf{l}_{1}, \mathbf{l}_{2}\right)$ (Fig. 10). From the homography $\mathbf{H}_{21}^{\pi_{1}}$ induced by $\pi_{1}$, we can obtain the corresponding of this line in the second image as $\overline{\mathbf{l}}_{2}=\left(\mathbf{H}_{21}^{\pi_{1}}\right)^{-T} \mathbf{l}_{1}$. The intersection of lines $\mathbf{l}_{2}$ and $\overline{\mathbf{l}}_{2}$ is the projection of the point $\mathbf{x}$ in the second image. In the same way, the process is repeated with another line, $\mathbf{n}$. From the intersection of lines $\mathbf{n}_{2}$ and $\overline{\mathbf{n}}_{2}=\left(\mathbf{H}_{21}^{\pi_{1}}\right)^{-T} \mathbf{n}_{1}$ the projection of the point $\mathbf{y}$ in the second image is obtained.

Points $\mathbf{x}$ and $\mathbf{y}$ belong to both planes $\pi_{1}$ and $\pi_{2}$. The line passing through the projection of these points $\mathbf{m}_{2}$, is the projection of the intersection of the planes. Therefore, as this line belongs to $\pi_{2}$, it can be used as the fourth line to compute the second homography.

Summarizing, each line gives a point belonging to the intersection of the planes. With two lines the intersection line is determined. In our case, three lines are available and therefore, three points define the intersection line. As the line will not pass exactly through these points due to image noise a least squares solution is used for computing this line. 
This method presents degeneracies if one of the other three lines is the intersection line of both planes, or if the obtained line is parallel or intersects in the same point with the two other lines.

Working with lines give us a stronger constraint than using points when we have two homographies and we want to compute the third. The method can be applied twice obtaining two intersection lines, and it only needs two initial matched lines to compute the third homography. If we work with points, we need three points again, because the epipole is unique for the image pair. This difference between points and lines is because less than three points do not define a spatial plane, while two lines are enough.

\section{Computing Through the Homology}

In the previous section, we have described a geometric method to compute a second homography $\mathbf{H}_{21}^{\pi_{2}}$ when a first homography $\mathbf{H}_{21}^{\pi_{1}}$ is known and three matched points or lines belonging to a second plane $\pi_{2}$ are available. Next, another alternative method to compute a second homography is presented. The main idea is the use of the planar homology, which is a well-known model from two planes projected in two views.

A planar homology is a plane projective transformation with five degrees of freedom. It has a line of fixed points, called the axis, and a fixed point not on the line, called the vertex (Fig. 2). Planar homologies arise naturally in an image when two planes related by a perspectivity in 3-space are imaged [9].

The projective transformation representing the homology can be parameterized directly [10 in terms of the vector representing the axis $\mathbf{a}$, the vector representing the vertex $\mathbf{v}$, and the characteristic cross-ratio $\mu$ as $\mathbf{H}=\mathbf{I}+(\mu-1) \frac{\mathbf{v} \mathbf{a}^{T}}{\mathbf{v}^{T} \mathbf{a}}$, being $\mathbf{I}$ the identity matrix. It can also be parameterized [5] as $\mathbf{H}=\mathbf{I}+\mathbf{v} \mathbf{a}^{T}$, which implies $\mu=\mathbf{v}^{T} \mathbf{a}+1$. The cross ratio $\mu$ is formed by corresponding points, the vertex and the intersection of their join with the axis, and it is an invariant of the homology.

A homology has two unary eigenvalues, the third eigenvalue coincide with the cross ratio $\mu$. Eigenvectors of the homology associated to double unary eigenvalue span the axis a, and the other eigenvector, associated to eigenvalue $\mu$, give us the vertex $\mathbf{v}[5]$.

From two homographies a homology can be computed as $\mathbf{H}=\mathbf{H}_{21}^{\pi_{2}}\left(\mathbf{H}_{21}^{\pi_{1}}\right)^{-1}$. First homography $\mathbf{H}_{21}^{\pi_{1}}$ has already been obtained and we want to compute second homography $\mathbf{H}_{21}^{\pi_{2}}$. The main idea in this second method is to determine a homology using the available features. A homology can be computed from three matched features since it has five degrees of freedom and each feature gives two constraints.

We can map the point $\mathbf{p}_{1}$ (Fig. 2a) in the second image through the first homography $\mathbf{H}_{21}^{\pi_{1}}$ using $\overline{\mathbf{p}}_{2}=\mathbf{H}_{21}^{\pi_{1}} \mathbf{p}_{1}$. So, we obtain three matched points $\left(\mathbf{p}_{2}, \overline{\mathbf{p}}_{2}\right)$, $\left(\mathbf{q}_{2}, \overline{\mathbf{q}}_{2}\right)$ and $\left(\mathbf{r}_{2}, \overline{\mathbf{r}}_{2}\right)$. Since the homology is $\mathbf{H}=\mathbf{H}_{21}^{\pi_{2}}\left(\mathbf{H}_{21}^{\pi_{1}}\right)^{-1}$, we have for the first match $\mathbf{p}_{2}=\mathbf{H} \overline{\mathbf{p}}_{2}$ and so forth. From the three matches, the vertex $\mathbf{v}$ and the axis a are obtained geometrically, the cross ratio is computed too, and from 


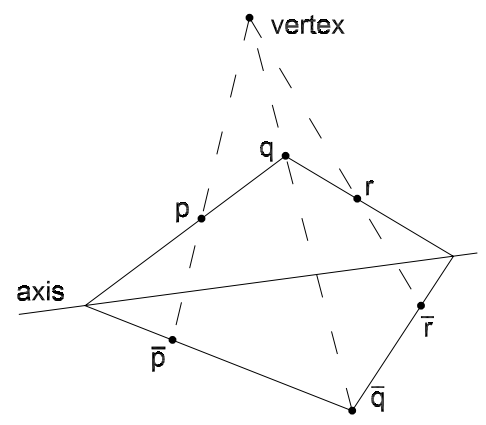

(a)

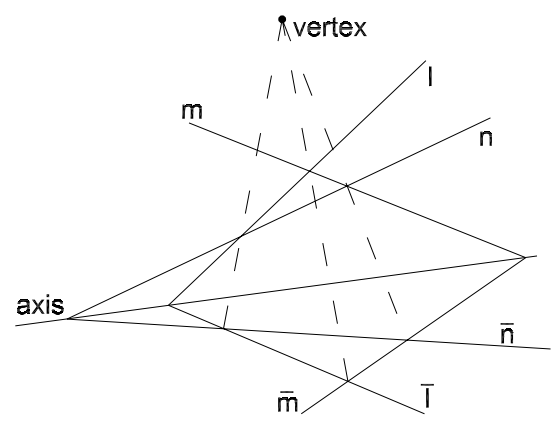

(b)

Fig. 2. Correspondences to compute a planar homology from points (a) or lines (b)

them the homology is computed using the previous parameterization. Finally, we can work out the value of the second homography from $\mathbf{H}_{21}^{\pi_{2}}=\mathbf{H H}_{21}^{\pi_{1}}$.

The process is similar using lines (Fig. 2 $\mathrm{b}$ ). The mapping of line $\mathbf{l}_{1}$ in the second image through $\mathbf{H}_{21}^{\pi_{1}}$ is carried out using equation $\overline{\mathbf{l}}_{2}=\left(\mathbf{H}_{21}^{\pi_{1}}\right)^{-T} \mathbf{l}_{1}$. From here, three matches are obtained: $\left(\mathbf{l}_{2}, \overline{\mathbf{l}}_{2}\right),\left(\mathbf{m}_{2}, \overline{\mathbf{m}}_{2}\right)$ and $\left(\mathbf{n}_{2}, \overline{\mathbf{n}}_{2}\right)$. Finally, second homography is computed as in the previous case.

\section{Experimental Results}

The proposed approach has been experimentally validated using synthetic data and real images. In all the experiments, the first homography has been automatically obtained and subsequently fixed, in order to avoid its variability (due to RANSAC) to affect the behavior of the computation of the second homography, which is analyzed.

The second homography is computed using all the methods presented: The general method of four features, the three features method and through the homology. The experiments are carried out with points and lines.

With real images the matches are automatically obtained for points and lines. In the case of points, they are extracted by Harris corner detector and matched by correlation and relaxation algorithms [11. The process of extract and match lines, which is based in geometric and brightness parameters, is explained in [7. The images of a house and a college with the matched features are shown in Fig. 3. The synthetic scene consists of random points in one case and lines on the other. These virtual features have white noise of $\sigma=0.3$ pixels and are distributed in three perpendicular planes.

Several criteria can be used to measure the accuracy of the obtained homography. We measure the first order geometric error computed as the Sampson distance [8] for a set of 20 matched points manually extracted and matched. Each experiment is repeated 100 times and the mean and median error is shown in Table 1. The accuracy obtained with all the methods is similar, demonstrating the validity of the proposed approach. The mean is always a little higher than 


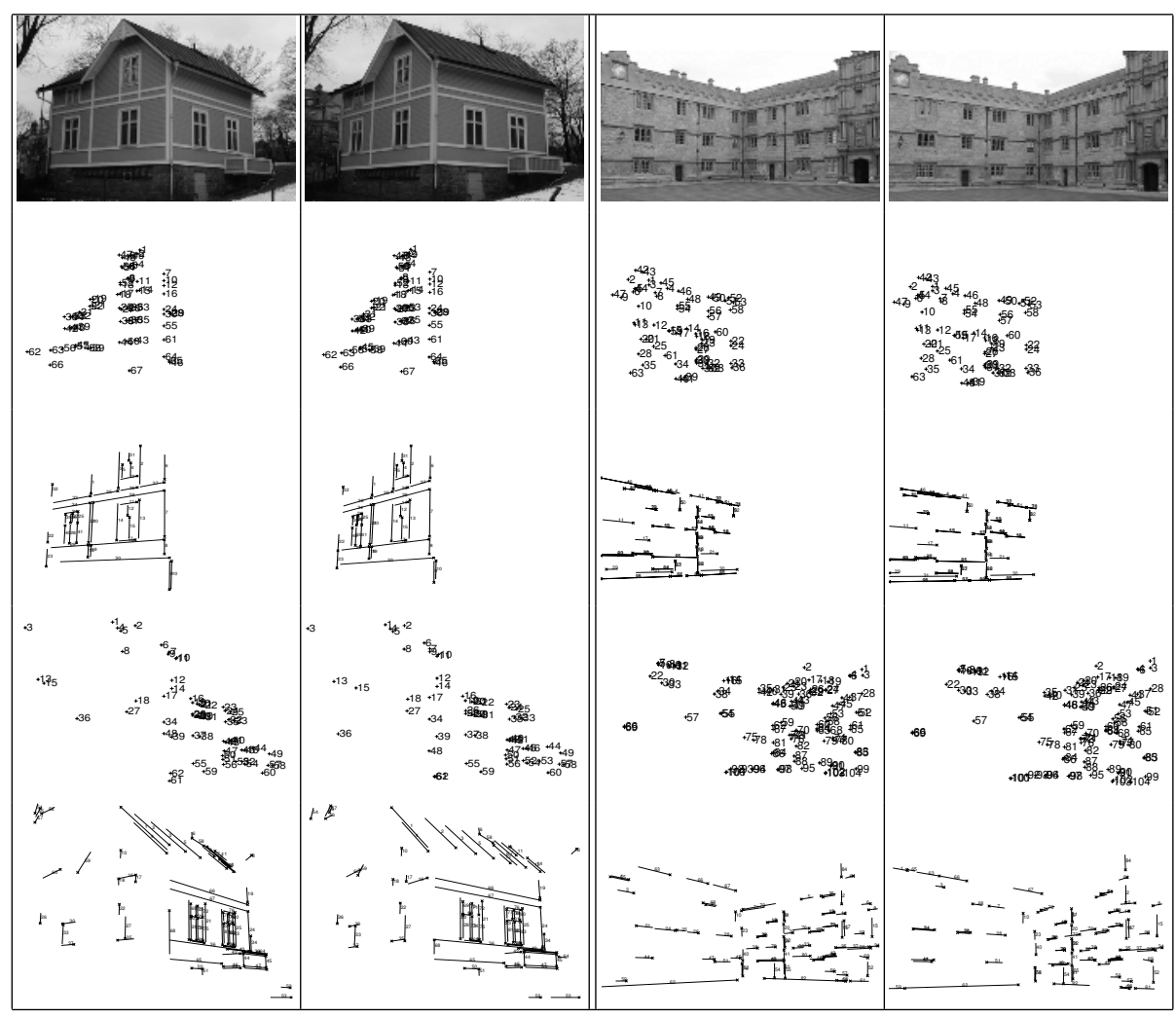

Fig. 3. Images of the house and college (1st row). Matches corresponding to the first homography with points (2nd row) and lines (3rd row). Putative matches available to search the second homography with points (4th row) and lines (5th row). Original images from KTH (Stockholm) and VGG (Oxford)

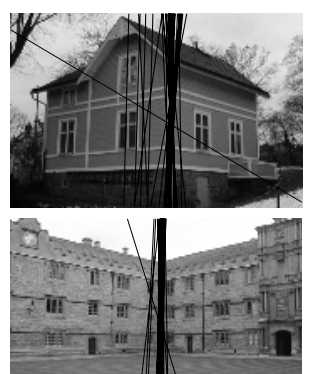

(a)

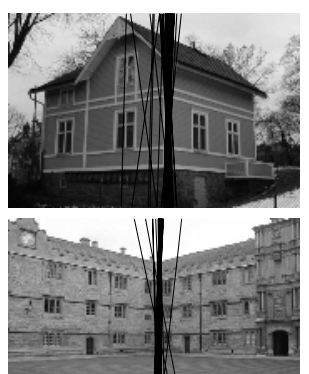

(b)

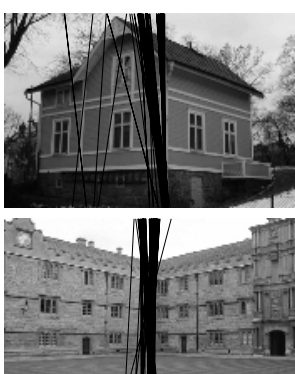

(c)

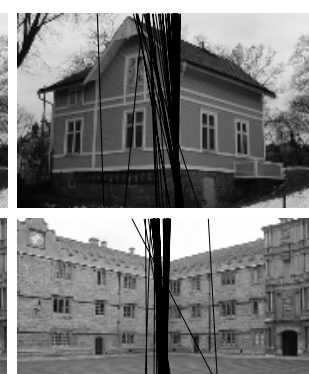

(d)

Fig. 4. Intersection of the planes through the eigenvalues of the homology. Lines corresponding to 100 executions are represented using 4 points (a); 3 points (b); 4 lines (c) and 3 lines (d) 
Table 1. Sampson distance of the second homography for 20 points manually matched. We show in 100 repetitions the mean and median error and the mean execution time. Results are obtained using four points (4p) or lines (4l); three points (3p) or lines (3l); and through homology with three points $(3 \mathrm{p}+\mathrm{H})$ or lines $(3 \mathrm{l}+\mathrm{H})$

\begin{tabular}{|c|l||c|c|c||c|c|c|}
\hline & $4 \mathrm{p}$ & $3 \mathrm{p}$ & $3 \mathrm{p}+\mathrm{H}$ & $4 \mathrm{l}$ & $3 \mathrm{l}$ & $3 \mathrm{l}+\mathrm{H}$ \\
\hline \hline \multirow{2}{*}{$\begin{array}{c}\text { Synthetic } \\
\text { data }\end{array}$} & Mean error (pixels) & 0.538 & 0.554 & 0.531 & 0.537 & 0.515 & 0.427 \\
\cline { 2 - 8 } & Median error (pixels) & 0.542 & 0.559 & 0.532 & 0.541 & 0.526 & 0.407 \\
\cline { 2 - 8 } & Mean time (seconds) & 6.94 & 1.94 & 1.66 & 4.21 & 1.50 & 1.43 \\
\hline \hline \multirow{2}{*}{$\begin{array}{c}\text { Images } \\
\text { of the } \\
\text { house }\end{array}$} & Mean error (pixels) & 1.230 & 1.191 & 1.206 & 1.257 & 1.591 & 1.576 \\
\cline { 2 - 8 } & Median error (pixels) & 1.168 & 1.171 & 1.162 & 1.134 & 1.099 & 0.821 \\
\cline { 2 - 8 } & Mean time (seconds) & 3.50 & 1.48 & 1.29 & 4.24 & 1.74 & 1.58 \\
\hline \hline \multirow{2}{*}{$\begin{array}{c}\text { Images } \\
\text { of the } \\
\text { college }\end{array}$} & Mean error (pixels) & 0.981 & 0.942 & 0.933 & 0.747 & 0.737 & 0.718 \\
\cline { 2 - 8 } & Median error (pixels) & 0.854 & 0.755 & 0.782 & 0.789 & 0.724 & 0.740 \\
\cline { 2 - 8 } & Mean time (seconds) & 4.08 & 1.43 & 1.48 & 4.71 & 0.92 & 1.15 \\
\hline
\end{tabular}

the median, this is because the presence of some spurious iterations. Sampson distance obtained with the four feature method is similar to the three features method, but it needs more iterations to get this similar result. Both methods of three features and using the homology can be used either because the obtained results are nearly equal.

There is almost no differences between errors obtained using points or lines. Although it depends on the extracted and matched features. In our experiments, the initial number of matches is similar for points and lines, and they are homogeneously distributed over the images. The use of lines instead of points has some advantages, mainly in man made environments. Straight lines can be accurately extracted in noisy images, they capture more information than points and they may be matched where partial occlusions occur.

Table 1 also shows the mean execution time of the iterations. One of the advantages of the three features method is that it consumes less time than using four features. This is because the number of samples necessary to avoid outliers with a high probability is exponential in the size of the sample set.

The epipole and the intersection of the planes can also be computed from the homology. The epipole is the eigenvector corresponding to the non-unary eigenvalue and the other two eigenvectors define the intersection line of the planes. In Fig. 4 the intersection line of the planes is shown for 100 repetitions. Only in a small percent of cases it is wrong, which is coherent with the probability of fail assumed in the robust method. Besides, in the same way that the features on the first plane have been automatically segmented (second and third row of Fig. 3), the method gives a robust selection of the features on second plane [1].

\section{Conclusions}

We have presented a method to compute a second homography from three points or lines in an image pair. In other works, each homography is computed indepen- 
dently of the others, and four features are needed. But once a first homography is obtained we can take advantage of the constraints imposed to determine another homography.

Experiments have been carried out in an automatic way using points and lines as features. Although the use of points is more common, lines has proven to give similar results, having advantages in man made environments.

The main advantage of this approach is the computing time reduction for the second homography. This is due to the use of a smaller size of the sample, which is three instead of four. This is useful to improve the performance of real time applications. Besides, the accuracy of the obtained homographies with the three features method is as good as using four.

\section{References}

1. Luong, Q., O.D.Faugeras: Determining the fundamental matrix with planes: Unstability and new algorithms. In: In Proc. Conference on Computer Vision and Pattern Recognition. (1993) 489-494

2. Vincent, E., Laganière, R.: Detecting planar homographies in an image pair. In: Proc. Second International Symposium on Image and Signal Processing and Analysis. (2001) 182-187

3. Shashua, A., Avidan, S.: The rank 4 constraint in multiple $(\geq 3)$ view geometry. In: Proc. European Conf. Computer Vision, St. Louis, Missouri (1996) 196-206

4. Zelnik-Manor, L., Irani, M.: Multiview subspace constraints on homographies. In: Proc. Int. Conf. Computer Vision, St. Louis, Missouri (1999) 710-715

5. Zelnik-Manor, L., Irani, M.: Multiview constraints on homographies. IEEE Transactions on Pattern Analysis and Machine Intelligence 24 (2002) 214-223

6. Pellejero, O., Sagüés, C., Guerrero, J.: Automatic computation of fundamental matrix from matched lines. In: Current Topics in Artifical Intelligence, LNCSLNAI 3040. (2004) 197-206

7. Guerrero, J., Sagüés, C.: Robust line matching and estimate of homographies simultaneously. In: IbPRIA, Pattern Recognition and Image Analysis, LNCS 2652. (2003) 297-307

8. Hartley, R., Zisserman, A.: Multiple View Geometry in Computer Vision. Cambridge University Press, Cambridge (2000)

9. Van-Gool, L., Proesmans, M., Zisserman, A.: Grouping and invariants using planar homologies. In: Workshop on Geometrical Modeling and Invariants for Computer Vision. (1995)

10. Criminisi, A., Reid, I., Zisserman, A.: Duality, rigidity and planar parallax. In: European Conference on Computer Vision, LNCS 1407. (1998) 846-861

11. López-Nicolás, G., Sagüés, C., Guerrero, J.: Automatic matching and motion estimation from two views of a multiplane scene. In: IbPRIA, Pattern Recognition and Image Analysis. (2005) To appear, LNCS. 